\title{
La dimensión axiologizante de la publicidad. Análisis del papel modalizador del texto publicitario desde la perspectiva de A. J. Greimas
}

\section{The Axial Dimension of Advertising. Analysis of the Advertising Text Modeling Role from thePerspective of A. J. Greima}

\section{Vladimir Sánchez Riaño (Colombia)}

Universidad Jorge Tadeo Lozano

Correo: vladimir.sanchez@utadeo.edu.co

\section{Resumen}

La publicidad es en sí una estructura narrativa que se modaliza (se realiza, modifica y al mismo tiempo transforma el estado de grupos de consumidores) a partir de la comunicación, con el objetivo de informar, distinguir (hacer reconocible), posicionar y reposicionar productos, bienes, servicios, personas o ideologías.

\author{
Abstract \\ Advertising is by itself a narrative structure \\ that is modeled (performed, modified, \\ and at the same time, transforms \\ the status of consumer groups) from \\ communication, in order to inform, \\ distinguish (make visible), position, and \\ reposition products, goods, services, \\ people, and ideologies. \\ This way, this article aims to apply some
}

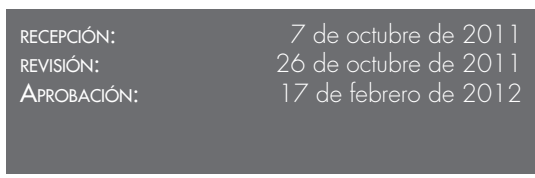

PARA CITAR ESTE ARTíCulo / tO CITE this ARTICLE

Sánchez Riaño, V. La dimensión axiologizante de la publicidad. Análisis del papel modalizador del texto publicitario desde la perspectiva de A. J. Greimas. Poliantea 8 (14), $11-30$ 
En este orden de ideas, el presente artículo busca aplicar algunos postulados de la propuesta de narratividad de Greimas a textos publicitarios, en un primer momento por medio de un procedimiento descriptivo sobre la base del hacer de la publicidad, y en un segundo momento, del análisis a un caso concreto de publicidad en un comercial que sirva como objeto para revisar la aplicación anunciada. Esta aplicación tiene por objetivo investigar el proceso de axiologización o vertimiento de valor que realiza la publicidad en objetos, productos, servicios, personas o ideologías.

Palabras clave: publicidad, valor, modalización, narratividad, texto. principles of the narrative proposal of Greimas on advertising books through a descriptive procedure (based on advertising), and through the analysis of a specific advertising case (used as an object to review the application formerly mentioned). This application is designed to analyze the process of adding value carried out by advertising with respect to objects, products, services, people, or ideologies.

Keywords: Advertising, value, modeling, narrative, text. 


\title{
La dimensión axiologizante de la publicidad. Análisis del papel modalizador del texto publicitario desde la perspectiva de A. J. Greimas
}

\begin{abstract}
Vladimir Sánchez
Profesor investigador de tiempo completo y coordinador del Área de Investigación del Programa de Publicidad de la Facultad de Ciencias Humanas, Arte y Diseño de la Universidad Jorge Tadeo Lozano. Filósofo y especialista en Docencia Universitaria de la Universidad Santo Tomás. Magíster en Estudios Políticos por la Universidad Javeriana y magíster en Semiótica por la Universidad Jorge Tadeo Lozano. Se ha desempeñado como profesor en las áreas de Semiótica, Sociología y Publicidad, Teorías de la Comunicación e Investigación durante los últimos catorce años en prestigiosas universidades de Bogotá.
\end{abstract}

\section{Introducción}

El objetivo de Greimas consiste, por lo tanto, en encontrar estructuras de la narrativa transversales a los diversos géneros textuales, en esta perspectiva, ha elaborado el concepto de "narratividad" y lo ha colocado al nivel seminarrativo, o, a un nivel suficientemente abstracto y profundo de modo que garantiza la universalidad.

Indiferente, por lo tanto, a las múltiples realizaciones expresivas de superficie $[. .$.$] Greimas admite una organización narrativa subyacente a la$ manifestación, y la considera una especie de competencia que aprovisiona a cualquier persona de la capacidad bien sea de reconocer y de producir textos. Y, de hecho, al nivel inmanente (unido de un modo inseparable a su esencia) de la generación del sentido que Greimas asume el concepto de la narratividad, como un tipo de trancón estructural común a cada texto,

\footnotetext{
El presente artículo de reflexión hace parte del ámbito teórico de la investigación, financiada por la Universidad Jorge Tadeo Lozano, denominada "La publicidad como espejo de la sociedad. Lectura sociosemiótica de algunos estereotipos culturales de Colombia a partir de anuncios publicitarios gráficos de las décadas de los cincuenta, sesenta y setenta", realizada por el Grupo Publicidad: Sociedad, Cultura y Creatividad, registrado en Colciencias en categoría C, código COL 0052199
} 
cualquiera que sea la naturaleza [1] (Magli, 2009 [2004],pp 68-69).

En realidades un modo diferente de entender la producción del sentido [...] La narratividad para Greimas, es por lo tanto una especie de gramática que, al nivel inmanente del sentido, articula las estructuras universales del imaginario.

Aunque en ocasiones desdeñada por la crítica social y estética, la publicidad, y más precisamente sus productos comunicativos (campañas, piezas y pautas), puede entenderse como ciertos tipos de textos narrativos en los que se realiza de forma cotidiana, constante y quizás inadvertida, el esquema narrativo de base y transversal a cualquier texto. Por ello es posible aplicársele la hipótesis greimasiana, según la cual la narratividad se constituye en un modelo lo suficientemente universal para comprender el sentido del texto.

Ahora bien, desde una perspectiva amplia puede decirse que estos tipos de textos son: "estrategias comunicativas que buscan generar en sus destinatarios una respuesta interpretativa de carácter favorable frente a un bien, servicio, producto, persona, política o ideología" (Sánchez, 2008, p. 380). En esa búsqueda es posible sostener que la publicidad de suyo es una estructura narrativa que se modaliza (se realiza y al mismo tiempo transforma el estado del target $)^{2}$ a partir de la comunicación, con el objetivo de informar, distinguir (hacer reconocible), posicionar y reposicionar productos, bienes, servicios, personas o ideologías.

De esta forma el objeto de valor propuesto por el texto publicitario ${ }^{3}$ se construye gracias a una sobremodalización o transformación de los enunciados descriptivos presentes en ella. En este sentido la publicidad adquiere su valor y su estatus social $y$, por supuesto, económico, gracias a que ella es el medio (objeto modal) para alcanzar un fin determinado y buscado por el cliente o anunciante. De modo que en un estado de disjunción del target frente al objeto de valor propuesto en el que, o no se tiene conocimiento, o no se distingue, o no se valora, o se ha perdido el valor frente al producto, bien, servicio, ideología o persona, el fin del texto publicitario sea lograr que un

2 Término utilizado para designar al sujeto individual o colectivo que es previsto como lector modelo o destinatario posible del mensaje (cfr. Eco, 1979, cap. 3).

3 De aquí en adelante se usará de forma genérica la expresión "texto publicitario" para hacer referencia a cualquier tipo de pieza publicitaria (comercial, cuña, pauta, exterior, nuevos medios, etcétera). 
sujeto (individual o colectivo), entre en conjunción con el objeto de valor propuesto y así tenga conocimiento de él, lo distinga de otro tipo de valores y de objetos, lo valore, o recupere cierto valor perdido en el producto, el bien, el servicio, la ideología o la persona.

Así las cosas, puede decirse que la publicidad es un objeto modal que sirve como medio para alcanzar un objeto de valor, que cambia dependiendo de si se ve desde la óptica del anunciante (advertir, distinguir, posicionar, reposicionar su producto), ${ }^{4}$ de la agencia que realice la campaña, del publicista o grupo de publicistas que la hacen, del target que la recepciona o del analista que la interpreta y valora. Sin embargo, al mismo tiempo ella se sirve de la comunicación como objeto modal para alcanzar su cometido, con lo cual (y retomando lo arriba anunciado), en la publicidad se realiza de forma clara una sobremodalización por vía de la manipulación (no en el sentido peyorativo del término, sino en el sentido técnico dado por Greimas como el hacer-hacer del programa

4 En adelante se utiliza la palabra "producto" de forma genérica para hacer referencia a: producto, bien, servicio, persona e ideología. narrativo), la competencia, el resultado y la sanción.

Ahora bien, la narración y en concreto los programas narrativos, son sistemas semióticos que se manifiestan en una materia expresiva, que se evidencia en diferentes tipos de textos, entendidos en el sentido de Eco como "un producto cuya suerte interpretativa debe tomar parte de su propio mecanismo generativo" (Eco, 1979, p. 79). Por ello al acercarse a estos dispositivos es necesario comprender la manera como se dan las estructuras puramente narrativas que constituyen el modelo narrativo abstracto, pero también, las estructuras discursivas que dan cuenta del nivel expresivo particular y la manera como las primeras se articulan en ella.

En este orden de ideas la publicidad y en especial el texto publicitario se puede asumir como un dispositivo objeto de estudio en el que se pueden distinguir las estructuras narrativas y las estructuras discursivas arriba planteadas. Pero, ¿cómo aplicar la propuesta de narratividad de Greimas a la estructura narrativa presente en el texto publicitario? En lo que sigue se buscará dar respuesta a este interrogante de acuerdo con la hipótesis de que 
la estructura narrativa subyacente a todo proceso publicitario se constituye de la siguiente manera: un sujeto (publicista) busca que un sujeto en disjunción con un objeto de valor, entre en conjunción con ese mismo objeto por medio de la identificación o construcción de nuevos elementos de valor. El esquema quedaría así:

$$
\mathrm{S}_{1} \rightarrow \mathrm{S}_{2} \cup \mathrm{Ov} \rightarrow \mathrm{S}_{2} \cap \mathrm{Ov}
$$

En términos generales, el presente artículo plantea la hipótesis de que el publicista, por medio de distintas modalizaciones, busca que el target que está en disjunción con el producto, entre en conjunción con él, a partir de procesos de modalización virtualizante (querer / deber) y actualizante (saber y poder).

Para sustentar la hipótesis anterior el presente artículo se desarrollará en: 1) traslape ${ }^{5}$ de la teoría de Greimas con el programa narrativo de la publicidad; 2) análisis y proyección a un caso concreto; 3 ) conclusiones analíticas.

5 Utilizo aquí la palabra traslape en lugar de aplicación porque en el primer momento lo que se busca es justamente cubrir la teoría con las lógicas narrativas de los textos publicitarios.

\section{Horizonte teórico}

En 1983 Greimas plasmó su propuesta de semiótica narrativa en Del sentido II, en la que propone la noción de narratividad como una suerte de tronco común a cualquier tipo de texto. Para ello desarrolla el concepto de objeto de valor y modalización como ejes desde los cuales se articula la universalidad (en el sentido de su aplicabilidad a cualquier texto) de la propuesta de semiótica narrativa y con ello del paso de la narratología a la narratividad. Al respecto Magli (2009, p. 69) sostiene: "es en realidad una forma diferente de entender la producción de sentido [...] La narrativa de Greimas, es una especie de gramática, en el sentido inmanente, articula las estructuras universales del imaginario".

En este sentido la conceptualización sobre los objetos de valor en la semiótica narrativa de Greimas se constituye en una discusión de primer orden, pues el objeto de valor es casi una condición necesaria para la agenda narrativa, en el hacer publicitario el objeto de valor va más allá del objeto en sí, pues este se carga axiológicamente cuando mediante de la acción publicitaria se da un vertimiento semántico o atribución de valores previamente definidos. 
Así, en el comercial de un ban$\mathrm{CO}^{6} \mathrm{o}$ entidad financiera como un real existente, en el que el concepto comunicativo o promesa básica es creer, dicho real existente puede estar vertido de valores cuando por medio de la publicidad se le carga con el diferencial creer como principio para lograr las cosas; dice el texto del comercial:

Cómo serían de diferentes las cosas si el que tuvo la idea de iluminar el mundo no hubiera creído en ella [...] Cómo habría cambiado la vida si aún no pudiéramos medir el tiempo; el mundo está hecho de ideas [...] creer por naturaleza es creer que todo es posible: Banco de Occidente.

Como se ve, el objeto de valor no es el banco en sí, sino el valor agregado que implica la confianza que otorga el banco a sus clientes, confianza que a su vez deben tener los clientes en él.

En efecto, desde la perspectiva greimasiana, el objeto del deseo en cuanto portador de un valor deseable por un sujeto, se constituye en la excusa narrativa que da origen a la fábula y con ella a la disposición

6 Cfr. comercial en: http://www.youtube. $\mathrm{com} /$ watch $\mathrm{v} v=\mathrm{N} 5 \mathrm{u} 6 \mathrm{pa} 4 \mathrm{C} 32 \mathrm{c}$. del discurso. Ahora bien, el objeto en sí no tiene un valor, solo ratifica una existencia o su realidad. En este caso la entidad financiera sobre la que se da el vertimiento de valores que permite convertir un simple objeto existente (el banco) en un objeto de valor (la confianza), convierte así a la confianza en el objeto deseado.

Desde la perspectiva de los cuentos maravillosos, Greimas propone la categoría de objetos mágicos para iniciar el análisis, entendidos como objetos que "una vez puestos a disposición del héroe o del antihéroe, les ayudan de diferentes maneras e incluso, a veces, les sustituyen en la búsqueda de valores" (Greimas, 1983, p. 22). Estos objetos - dice Greimas- pueden distinguirse en bienes o servicios. Los primeros, consumibles y atesorables, con los cuales el héroe logra realizar sus cometidos; por ejemplo, el poseer el antitranspirante adecuado puede hacer que el sujeto logre establecer una realización de apareamiento con la hembra de su especie, como sería el caso de la publicidad de desodorantes AXE. Por otro lado estarían los servicios no explícitamente desarrollados y que tienen que ver con ciertas capacidades o potencialidades del héroe que se convierten igualmente en coadyuvantes para el logro de sus 
cometidos: tener la tarjeta de crédito adecuada (en el caso del comercial de Master Card) le permite desenvolverse adecuadamente en un ambiente en cuyo caso el objeto de valor no es la tarjeta en sí, sino el cubrimiento mundial que esta le ofrece.

Ya en la reflexión sobre el objeto y el valor, podría decirse que el objeto adquiere mayor valor en la medida en que genera mayor resistencia frente al sujeto de deseo a la hora de alcanzarlo, y esta resistencia se constituye en tanto que el objeto comporta diversos tipos de dimensiones, como: dimensiones figurativas y casi constitutivas de él (en términos fisiológicos); dimensiones fácticas en cuanto variables de diferenciación frente a otros objetos; y por último, dimensiones funcionales en el ámbito práctico y mítico que otorgan prestigio. Piénsese en ciertos comerciales para automóviles como Peugeot, Audi o Mercedes, en los que el objeto de valor surge tras el vertimiento axiológico al objeto factual, de prestigio y estatus. En relación con la adquisición del valor, Greimas sostiene:

Cuando alguien, por ejemplo, en nuestra sociedad actual, se presenta como adquisidor de un automóvil, quizá no es tanto el coche como objeto lo que desea adquirir, sino, en primer lugar, un medio de desplazamiento rápido, [...] lo que compra a menudo es también un poco de prestigio social o un sentimiento de potencia más íntimo (Greimas, 1989, p. 25).

Así pues, para Greimas el objeto en sí como categoría narrativa no tiene sentido, sino en la medida en que el objeto comporta una serie de valores de carácter social o cultural que se convierten en el tensor más fuerte en el procedimiento ideal o quizá metafórico de resistencia del objeto frente al sujeto que lo desea:

Hablar, por tanto, de objetos en sí mismos no tiene sentido, e incluso el tratamiento taxonómico de una clase de objetos [...] no opera más que con categorías sémicas, es decir, solo con los valores: siempre queda una distancia entre el paquete de semas que organiza metalingüísticamente la representación del sillón y el lexema terminal sillón (Greimas, 1989, p. 26).

Como se deduce, el valor del objeto se encuentra en íntima relación con el sujeto, pues en esta relación se vierte el valor sobre el objeto gracias a los deseos del sujeto y se semantiza de este modo el enunciado en sí:

En la medida en que el enunciado elemental puede definirse como relación orientada que engendra sus 
dos términos resultantes (el sujeto y el objeto), el valor que se vierte en el objeto enfocado semantiza en cierto modo el enunciado entero (Greimas, 1989, p. 27).

El publicista construye su proceso de semantización, o si se quiere, de axiologización, de su objeto de valor, apelando a los deseos y anhelos de su target.

Ahora bien, la constitución del valor de los objetos puede entenderse en términos de valores objetivos o valores subjetivos en la medida en que los primeros se engendran o encarnan en relaciones del tener, mientras que los segundos se encarnan en relaciones del ser. Si se quiere, los primeros se asocian con los bienes y los segundos con los servicios, distinción planteada arriba. Así la diferencia entre una pieza publicitaria de carácter promocional con una de carácter mostrativo de cualidades consistiría en que en la primera el vertimiento de valores se da sobre las características del producto o el precio de él, mientras que en la segunda se da sobre sus cualidades. En el primer caso el objeto de valor se constituye en la usabilidad o economía del producto, mientras que en el segundo el valor se constituye en una abstracción como ventaja alcanzada por usarlo.
La narrativización —según Greimas - se concibe sobre la base del estatus semiótico del objeto y se estructura en el ámbito sintáctico del discurso, en el cual a partir de los niveles de conjunción y disjunción dados entre el sujeto y el objeto se construyen los enunciados. La realización del discurso no es más que la transformación que establece la conjunción o disjunción entre el sujeto y el objeto:

La disjunción, al ser la negación de la conjunción, no constituye la abolición de toda relación entre dos actuantes: de lo contrario, la pérdida de toda relación entre sujetos y objetos llevaría a la abolición de la existencia semiótica y devolvería los objetos al caos semiótico original. [...] La disjunción no hace sino virtualizar la relación entre el sujeto y el objeto, manteniéndola como un posibilidad de conjunción (Greimas, 1989, p. 33).

Ahora bien, el revestir de valor al objeto implica — según Greimasestablecer la relación entre el sujeto y el objeto, mientras que la pérdida del valor, por la circunstancia que fuere, se encarna en la abolición total de la relación con el sujeto y el objeto. Así, cuando en un comercial de seguros (en el que un niño rompe un enorme vidrio de una casa con 
su balón de fútbol y entra por el orificio causado por el accidente para recuperar su objeto, encontrándose con la sorpresa de que el vidrio ya está puesto, por la eficacia de la empresa aseguradora), ${ }^{7}$ lo que sucede es que el accidente y el vidrio roto como objeto de valor con el cual entró en disjunción el niño, se desfiguró y perdió toda relación, pues ahora la rapidez y eficacia con la que la empresa aseguradora soluciona estos casos entra en conjunción con el sujeto del target al que va dirigido.

El acto publicitario en sí está justamente fundado en estos procedimientos de axiologización que hacen que el producto empiece a tener vida social y cultural, pues más allá del nivel funcional de lo que se ofrece, se construye un objeto de valor que se comunica en el acto publicitario. De modo que el valor es lo adjudicado a un objeto para que se transforme en un objeto de valor con respecto a un sujeto, sobre la base de una primera disjunción, que debe ser resuelta haciendo entrar al sujeto en conjunción con el objeto de valor. Como se da en la famosa fórmula publicitaria: “ ¿aún no tienes

7 Cfr comercial en: http://www.youtube.com watch? $\vee=0 q$ VH_lWbNJA. la tecnología X? ¡Adquiérela con el producto Y!”.

Para que el programa narrativo se logre en su totalidad se apela también al objeto modal en el sentido de que un objeto buscado es medio para alcanzar el objeto de valor. Este objeto modal permite introducir la categoría de estado modal que genera la transformación y en últimas la entrada en conjunción del sujeto con su objeto de valor. Greimas definió cuatro tipos de estados modales: poder, saber, deber y querer.

Podría decirse que la modalización es la producción de un enunciado llamado modal que sobredetermina un enunciado descriptivo. Este proceso se logra cuando la categoría semántica de base se axiologiza por vía de la categoría tímica: ${ }^{8}$ "se diría, pues, que la aplicación de

8 Para Greimas la categoría tímica es de carácter primitivo y propioceptivo (formula el modo en el que un ser vivo se siente $y$ siente), se encuentra en la estructura profunda y permite articular el semantismo directamente ligado a la percepción que el ser humano tiene. Esta categoría se articula en euforia/disforia y es la base para el paso de las estructuras profundas a las superficiales o de los microuniversos semánticos a las axiologías. Así, la conversión de la categoría tímica del nivel profundo al superficial se da gracias a la actualización que hacen los sujetos a partir de los valores adoptados por ellos; este proceso es el que hemos denominado modalización o vertimiento de valor a los objetos. 
lo 'tímico" sobre lo 'descriptivo' ${ }^{10}$ transforma las taxonomías en axiologías" (Greimas, 1989, p. 108).

Ahora bien, el hecho de que el vertimiento semántico se dé en una categoría semántica constituyente de la estructura elemental, no asegura de suyo la constitución de la modalización, sino que requiere que una vez se haya dado la categorización, se dé también el paso de la taxonomía a la axiología, es decir, el procedimiento mediante el cual se sobredetermina el enunciado descriptivo gracias la apelación a la categoría tímica que articula el semantismo vinculado a la percepción de lo eufórico/disfórico, en relación con la constitución de valor. En términos concretos, se trataría del paso del objeto factual "tarjeta de crédito" al objeto de valor "prestigio o cobertura mundial", lo que convierte, al mismo tiempo, al objeto factual "tarjeta de crédito" en objeto modal, mediante el cual se alcanza el verdadero objeto de valor deseado y adquirido cuando se adquiere el producto; en palabras de Greimas:

Una categoría semántica puede ser axiologizada por la proyección en el cuadro que lo articula, de la

9 Comillas en el texto original.

10 Comillas en el texto original. categoría tímica cuyos términos contrarios son denominados /euforia/ vs. /disforia/. Se trata de una categoría "primitiva" [...] con cuya ayuda se trata de formular [...] el modo en que todo ser vivo, inscrito en un medio, se "siente" a sí mismo y reacciona frente a su entorno (Greimas, 1989, p. 106).

De modo que la transformación de las taxonomías en axiologías se da cuando se aplica lo tímico sobre lo descriptivo, con lo que se logra pasar de una jerarquía de unidades y elementos, entendidos como objetos semióticos, a una valorización de dichos objetos. Todo ello en función de procedimientos que pueden ser asociados con la competencia (modalización del ser al hacer), de la performance (modalización del hacer al ser), de la factitividad (modalización del hacer al hacer) y de la veridictividad (modalización del ser al ser). Así se pasa de la taxonomía a la axiología en lo virtualizante (querer- y deber-hacer), lo actualizante (poder-y saber-hacer) y lo realizante (hacer-ser).

Mientras que las modalizaciones asociadas con la performance, es decir, la modalización del hacer al ser, constituyen las modificaciones del estatus del ser del sujeto, las modalizaciones asociadas con lo veridictivo, 
esto es, del ser, al parecer pueden considerarse como modificaciones del estatus del objeto de valor.

Desde el punto de vista publicitario las primeras tienen como centro el sujeto o target, mientras que las segundas tienen como centro al objeto de valor. En primer lugar las modalizaciones del hacer hacia el ser construyen un programa narrativo consistente en asegurar la transformación del estatus del ser del sujeto cuando este (el target) alcanza el objeto de valor axiologizado en el objeto modal que adquiere. En segundo lugar las modalizaciones del ser al ser construyen un programa narrativo que consiste en presentar una transformación en el producto, de modo que este, como objeto modal, asegure al sujeto que la adquisición le lleva alcanzar su objeto de valor, a pesar de que antes no lo hacía y ello justamente por la transformación sufrida en él.

La conversión de los valores axiologizados que se da de la estructura profunda a la estructura superficial reside en la actualización de los valores adoptados por el sujeto manteniendo el estatus de los valores axiológicos. Greimas trata de explicar entonces cómo se da esa conversión de la estructura profunda a la superficial gracias a los procesos de modalización, que terminan por generar así procesos de significación en el recorrido generativo:

El espacio tímico que, a nivel de las estructuras abstractas, se supone representa las manifestaciones elementales del ser viviente en relación con su entorno [...] encuentra correspondencia a nivel más superficial, antropomorfo, del recorrido generativo, en el espacio modal que, al mismo tiempo que cubre el mismo lugar tópico, se presenta como una excrecencia y una sobrearticulación del primero (Greimas, 1989, p. 107).

El proceso en general se da gracias a la serie de subarticulaciones en el ámbito profundo que se antropomorfiza y se actualiza debido al proceso de modalización que se desarrolla gracias al vertimiento que se da en los cuatro términos modales del querer, del deber, del poder y el saber. Estos términos modales constituyen entonces el punto de partida de la construcción del mensaje publicitario, pues la axiologización del objeto de valor va a estar centrada en: el deseo, la necesidad, el prestigio o estatus y la competencia.

\section{Metodología}

En principio es pertinente anotar que el interés analítico de este artículo es 
proyectar los principales postulados de la propuesta de narratividad de Greimas al acto publicitario, inicialmente de forma general y descriptiva sobre la base del hacer de la publicidad y en un segundo momento en el análisis a un caso concreto de publicidad en un comercial que sirva como objeto para revisar la aplicación anteriormente anunciada. Por lo anterior el aporte del artículo consiste en un ejercicio de apropiación, proyección y ejemplificación de los postulados sobre la narratividad planteados por Greimas a un caso concreto de comunicación publicitaria. ${ }^{11}$

Así, para el caso particular de este artículo se hace el análisis de un comercial colombiano del producto precocido Harina Pan, con el cual se prepara el alimento típico llamado

11 Un desarrollo más sistemático de las categorías greimasianas en la publicidad se puede encontrar en Luis Sánchez Corral, quien ha llevado a cabo un desarrollo sistemático de las propuestas teóricas de Greimas, el cual debe considerarse como trabajo pionero en la constitución de una semiótica publicitaria, como semiótica específica. lgualmente son de reseñar los trabajos semiótico narrativos que al respecto han realizado autores como Jacques Fontanille, Claude Zilberberg, Jesús García Jiménez, Vicente Peña Timón (cfr. bibliografía). Sin embargo, no se pretende ni una discusión con estos autores, ni una ampliación de sus análisis, sino un análisis socioespacialmente ubicado en Colombia desde la fuente primaria, es decir, desde Greimas. arepa, el cual se trata de una circunferencia de harina de no más de $3 \mathrm{~mm}$ de espesor que se asa por ambos lados sobre una parrilla y normalmente se sirve en el desayuno. También se hace referencia al comercial de una entidad financiera - Banco de Occidente- $y$ a una serie de comerciales sobre el antitranspirante Axe, que, en el momento en que se escribió este artículo, se encontraban anunciados en la televisión colombiana. Para referenciar estos comerciales solamente se cita el lugar electrónico en el que se pueden observar, ya que no se trata de una indagación sobre el anunciante, la agencia y los medios que intervinieron en la producción y en la pauta de las piezas en cuestión.

Aunque el análisis se refiere a un comercial concreto, es claro que el modelo de programa narrativo puede ser aplicable a cualquier tipo de publicidad, ya sea cuña, anuncio, publicidad exterior o cualesquiera de las variantes de ella. Ahora bien, se eligió la categoría comercial por su confluencia de canales y de códigos, lo cual permite plantear que si el modelo puede funcionar en un texto audiovisual, fácilmente podría hacerlo en un tipo de publicidad simple en la que no se dé en una expresión pluricanal ni en un discurso pluricódigo. 


\section{Análisis y proyección a un caso concreto}

Para este análisis se utilizará un comercial de Harina Pan que en el 2011 se encontraba al aire en la televisión colombiana, este producto para preparar arepas se puede ver en la dirección http://www.youtube. com/watch?v=5hrEvPx9A7g, en un riel de comerciales con tiempo de reproducción comprendido entre 1:23-1:40. El comercial se construye a partir de la escena familiar de una madre joven y sus dos pequeños hijos, en la que aparece la voz en de fondo de una mujer joven que dice:

Lo que más me gusta del fin de semana es dedicárselo a mi familia, yo les dedico toda mi luz (detalle de la joven madre sonriente ofreciendo la arepa ya hecha, un hilo de mantequilla sobre ella emulando una sonrisa iluminada y con un primer plano en la margarina esparcida sobre ella), yo les regalo toda mi sonrisa (detalle de la arepa ofrecida a su hijo con una línea de margarina emulando una sonrisa) y les doy todo mi amor, porque con Harina Pan preparo las arepas más ricas, hechas con puro maíz (detalle de una mazorca tierna). Amor puro, puro maíz... es Harina Pan.

El programa narrativo del comercial está construido desde un proceso de modalización en el que a partir del hacer de la representación narrativa en la que se axiologiza el papel amoroso de la madre se busca transformar al ser, que en este caso sería el individuo del target, a partir de un vertimiento de valor en el que la harina es ya un objeto modal que permite la consecución del objeto de valor que es "el amor puro, el puro amor".

Así se da el paso de un enunciado del hacer que rige o transforma un enunciado de estado (familiar) en una representación que transforma y construye la situación familiar ideal cargada del valor: "amor, puro amor". Ahora bien, para hacer cualquier cosa se requiere ser capaz de saberla hacer, o lo que es lo mismo, se requiere la competencia, que hace que el ser modalice un hacer. Entonces lo importante no es realmente el hacer el producto como tal, sino el ser (madre) que sabe transformar el objeto modal para convertirlo en objeto de valor.

Con ello el programa narrativo parte desde la manipulación (hacerhacer) que busca orientar al sujeto, al hacer mediante unas acciones persuasivas o manipulantes para que este adquiera la competencia, la lleve a cabo en la representacióny sea sancionado, en este caso por su familia 
como juez, quien emite su juicio de aceptación y de amor a su madre, y se consolida así el programa narrativo.

En síntesis, desde el punto de vista del propósito del anunciante el comercial construye su programa narrativo al generar la manipulación en el target a partir de la presentación de su producto como objeto modal del hacer del ser (elaboración del producto como objeto modal), que le otorga la competencia al sujeto que desea alcanzar el objeto del deseo "amor, puro amor", y que es sancionado por su familia cuando esta es feliz. Ahora bien, el anunciante tiene su propio objeto del deseo, que es posicionar su producto (querer), lo cual se convierte en su manipulador, y aunque no tiene la competencia (el saber) para realizarlo, sí posee la capacidad (poder) de contratar al sujeto competente que construye el programa narrativo (representación) y juntos esperan la respuesta del target (sanción), que puede valorarse en términos de recordación, memorabilidad, decisión de compra y compra final del producto.

Como se ve, el objeto factual o real sobre el que versa el comercial, o spot, como real existente, es un producto precocido para preparar arepas. Sin embargo, el concepto comunicativo o promesa básica es el amor de la madre, de modo que el producto factual o real existente ha sido axiologizado al vertir el valor por medio del diferencial amor. Así entonces, el objeto de valor no es la harina para hacer arepas, sino el valor agregado al real existente: Harina Pan, que implica el amor de la madre y esposa que es feliz dedicándole su tiempo a su familia y presentándoles lo mejor que es su luz, su sonrisa, su amor, y las más ricas arepas, que se preparan con Harina Pan.

Por lo tanto, el objeto del deseo, en cuanto portador de un valor deseable por el sujeto destinatario del spot, se constituye en la excusa narrativa sobre la cual se construye la estructura o disposición del discurso, en el que más allá del producto real se lleva a cabo una sobredeterminación de él (Harina Pan) o modalización, al convertir el real existente (Harina Pan) en un objeto de valor (el amor), convirtiendo así la luz maternal, la sonrisa y sobre todo el amor, en el objeto deseado. Con ello el objetivo publicitario se determina de forma clara, al buscar el cambio de estado en el destinatario (en este caso madres de familia), de quienes se espera que entren en conjunción con el producto por vía de la asociación del amor familiar con un producto de 
alta calidad como lo es Harina Pan. De esta manera el producto se presenta como una especie de "objeto mágico" (categoría greimasiana planteada arriba) que se pone a disposición de la madre de familia, o heroína (en términos greimasianos). El producto presentado es un "bien" - no un servicio- con el cual ella alcanza sus deseos, en este caso la heroína al adquirir el producto ofrecido puede prepararle a su familia un desayuno formidable. Como se ve, el producto factual o real existente, al ser asumido como categoría narrativa, adquiere sentido en la medida en que el objeto comporta un valor familiar y personal como lo es el "amor filial". Dicho valor es entonces el tensor más fuerte en el procedimiento de resistencia del objeto frente al sujeto que lo desea y se constituye en el eje central del programa narrativo.

Como se decía (en la presentación de la teoría greimasiana), el valor del objeto se encuentra en íntima relación con el sujeto, pues en esta relación se vierte el valor sobre el objeto gracias a los deseos del sujeto. La semantización del objeto es lograda por el publicista al asociar los deseos $\mathrm{y}$ necesidades primitivas que subyacen en el interior de toda madre con los beneficios que se alcanzarían si se usara el producto anunciado. La modalización o constitución del valor del objeto se da entonces a partir de las relaciones del ser, en la medida en que dicha modalización se hace sobre las cualidades del producto, el cual es: amor puro... puro maíz. De esta forma el elemento articulador que hace que el destinatario entre en conjunción con el producto anunciado es el amor como objeto de valor.

Ahora bien, la modalización en el texto publicitario objeto de análisis se alcanza entonces apelando a la axiologización del producto por vía de la categoría tímica, en la cual se espera que el destinatario o miembro del target (en este caso madre de familia) genere euforia frente al producto, por medio de los sentimientos de amor, felicidad y regocijo familiar que - se supone- vibran en los estados primitivos de toda madre. De esta forma se logra una sobredeterminación del enunciado descriptivo Harina Pan gracias la apelación a la categoría tímica amor, que articula el semantismo vinculado a la percepción de lo eufórico/disfórico en relación con la constitución de valor. En suma, el programa narrativo del texto publicitario en cuestión permite el paso del objeto factual Harina Pan al objeto de valor amor, lo que 
convierte, al mismo tiempo, al objeto factual Harina Pan, en objeto modal, con lo cual se alcanza el verdadero objeto de valor deseado cuando se adquiere el producto.

\section{Conclusiones}

\section{Del programa narrativo publicitario y su modalización}

El programa narrativo de la publicidad puede ser descrito y normalizado (en el sentido de construcción metódica del mensaje) a partir de las propuestas teóricas planteadas por Greimas en su postura sobre la narratividad y la modalización presente en ella. Lo cual se daría así:

Transformación publicitaria: se da gracias a la oposición: aserción-negación; antes de una aserción hay una negación. Una transformación presupone un estado anterior, por ello la aserción tiene memoria, la campaña o pieza publicitaria busca esa transformación al vertir valores con los que construye el objeto del valor que le presenta al sujeto target de su acción.

Competencia publicitaria: comprensión de las condiciones socioculturales y semánticas en las que se da la realización del acto publicitario de modo que se logre a partir del hacer ser, que se dé el ser al modalizar al hacer. La competencia es del orden del ser, no del hacer, es anterior y previa a toda realización.

Representación: realización del acto publicitario en sí, lo que supone una competencia, es el hacer que modaliza al ser, siempre requiere una manipulación, que en el caso publicitario vendría dada por el interés o propósito que induce a la acción comunicativa de la publicidad.

Sanción: ser que modaliza el ser, valorable en términos de recordación, memorabilidad, decisión de compra y compra del producto. Esta sanción está inscrita en el esquema narrativo y ocupa las dimensiones cognoscitiva y pragmática, es ejercida por el destinador final o juez (target) y presupone una competencia absoluta en él; puede ser negativa (castigo) o positiva (recompensa), dependiendo de la eficacia del programa narrativo y con ello del vertimiento de valor del objeto modal al objeto de valor.

Manipulación: el mensaje publicitario de suyo tiene como punto de partida la manipulación, pues espera que mediante la transformación el sujeto que posiblemente se encuentra en disjunción con el producto entre en conjunción con él, gracias a vertir valores que lo constituyen como objeto de valor más allá del producto en sí. De este modo se trata de la acción 
de una persona sobre otras para que realicen algún programa narrativo. Es una estructura modal de tipo factitivo en la que un destinador-manipulador impulsa al destinatario hacia una posición y constituye el posible recorrido narrativo del destinatario.

\section{Del esquema narrativo publicitario y su realización}

El esquema narrativo debe ser entendido como el modelo general para dar cuenta de la organización de todos los discursos narrativos que constituyen el hecho publicitario en el cual se presenta la iteración de tres pruebas: 1) prueba calificante: competencia; 2) prueba decisiva: realización; y 3) prueba glorificante: sanción. Este modelo se revela en una organización sintagmática propia de todo esquema canónico. El esquema tiene tres recorridos: el recorrido narrativo del sujeto del hacer, el recorrido narrativo del manipulador y el recorrido narrativo de la sanción. Así, en el esquema narrativo de la publicidad se pueden distinguir tres recorridos narrativos:

- Recorrido del destinador-manipulador: aquí se da todo el programa narrativo del anunciante y su propósito publicitario constituido como objeto de valor, que no es el mismo objetivo de comunicación, pues con este último se busca alcanzar el propósito superior de la publicidad; en ese sentido, la comunicación es un objeto modal de la publicidad. En dicho aspecto la competencia es de la agencia que realiza el mensaje y constituye así la representación, mientras que la sanción viene del lado del juez-destinatario-target como respuesta a la acción.

- Recorrido del sujeto que representa: agencia-publicista que desarrolla su programa narrativo gracias a la manipulación dada por el cliente-anunciante. Posee la competencia (saber publicitario) que le permite llegar a la representación (hacer publicitario), con la cual espera una sanción favorable, no solo del destinatario-target, sino también del juez-sancionador-anunciante.

- Recorrido del destinatario juez: el individuo miembro del target que recibe el programa narrativo tanto del anunciante como del publicista y que es manipulado por los dos anteriores, tiene la competencia que le permite recibir y comprender el mensaje 
y con su actitud sanciona favorable o desfavorablemente, tanto al destinador-manipulador, como al sujeto que representa.

En síntesis, podría decirse que la narración publicitaria como agente en la transformación de estado construye una serie de programas narrativos que van desde el cliente $o$ anunciante y pasan por la agencia, los creativos, diseñadores y productores, hasta el target. Estos programas narrativos tienen su lugar de confluencia en el vertimiento de valor como artificio de la narratividad, en el que el objeto factual se transforma en un objeto de valor que debe ser deseable por cualquier destinatario o lector ideal al que vaya dirigida la comunicación.

\section{Referencias}

Eco, U. (1979). Lector in fabula. Barcelona: Lumen.

Fontanille, J. \& Greimas, A.J. (1994). Semiótica de las pasiones. De los estados de cosas a los estados de ánimo. México: Siglo XXI.

(2001). Semiótica del discurso. Lima: Fondo Editorial Univer- sidad de Lima, Fondo de Cultura Económica. (2004). Soma y sema. Figuras semióticas del cuerpo. Lima: Fondo Editorial Universidad de Lima.

Fontanille, J. \& Zilberberg, C. (2004). Tensión y significación. Lima: Fondo Editorial Universidad de Lima.

Greimas, A.J. (1966). Semántica estructural. Madrid: Gredos.

Greimas, A.J. \& Courtés, J. (1979). Semiótica. Diccionario razonado de la teoría del lenguaje, I. Madrid: Gredos. 1989 [1983]. Del sentido II. Madrid: Gredos.

Magli, P. (2004). Semiótica. Teoría, método, análisis. Venezia: Marsilio.

Peña, V. (1994). El programa narrativo como expresión del valor constitutivo del relato en el spot publicitario audiovisual. Tesis doctoral. Universidad Complutense, Madrid.

Sánchez, C. (1997). Semiótica de la publicidad. Madrid: Síntesis.

Sánchez, V. (2008). Semiosis y publicidad. Ensayos semióticos. Bogotá: Universidad Jorge Tadeo Lozano. 


\section{Referencias complementarias}

Barthes, R. (1990). La aventura semiológica. Barcelona: Paidós.

Cassirer, E. (1993). Antropología filosófica. Bogotá: Fondo de Cultura Económica.

Cruz Vélez, D. (1986). De Hegel a Marcuse. Bogotá: USTA.

Deledalle, G. (1996). Leer a Peirce hoy. Barcelona: Gedisa.

Eco, U. (1968/1999). Sección B. La mirada discreta, numeral 5. La estructura ausente. Barcelona: Lumen. (1973/2000). Signo. Barcelona: Lumen. (1976/2000). Tratado de semiótica general. Barcelona: Lumen.

Ferrer, E. (2002). Publicidad y comunicación. México: Fondo de Cultura Económica.

Groupe, U. (1987). Retórica general. Barcelona: Paidós.

(1992/1993). Tratado del signo visual: para una retórica de la imagen. Madrid: Signo e Imagen.
Klinkenberg, J.-M. (1996/2006). Manual de semiótica general. Bogotá: Universidad Jorge Tadeo Lozano. (2003). Claves cognitivas para una solución al problema del iconismo. DeSignis (4), 15-25.

Magli, P. (2009) [2004]. Semiótica. Teoría, método, análisis. Venecia: Marsilio.

Martín-Barbero, J. (1996). La televisión o el mal de ojo de los intelectuales. Número, 10, 37-42.

Morris, Ch. (1938/1985). Fundamentos de la teoría de los signos. Barcelona: Paidós.

O'Guinn, A. \& Semenik (1999). Publicidad. México: Thompson.

Ospina, W. (1994). El canto de las sirenas. Es tarde para el hombre. Bogotá: Norma.

Peirce, Ch. S. (1931-1958). CP Collected Papers. Ch. Hartshorne, P. Weiss y A.W. Burks (Eds.). (Vols. 1-8 C). Cambridge (Mass.): Harvard University Press. 